\title{
The stochastic distribution of available coefficient of friction on quarry tiles for human locomotion
}

\author{
Wen-Ruey Chang*, Simon Matz and Chien-Chi Chang \\ Liberty Mutual Research Institute for Safety, 71 Frankland Road, Hopkinton, MA 01748, USA
}

\begin{abstract}
The available coefficient of friction (ACOF) for human locomotion is the maximum coefficient of friction that can be supported without a slip at the shoe and floor interface. A statistical model was introduced to estimate the probability of slip by comparing the ACOF with the required coefficient of friction, assuming that both coefficients have stochastic distributions. This paper presents an investigation of the stochastic distributions of the ACOF of quarry tiles under dry, water and glycerol conditions. One hundred friction measurements were performed on a walkway under the surface conditions of dry, water and $45 \%$ glycerol concentration. The Kolmogorov-Smirnov goodness-of-fit test was used to determine if the distribution of the ACOF was a good fit with the normal, log-normal and Weibull distributions. The results indicated that the ACOF appears to fit the normal and log-normal distributions better than the Weibull distribution for the water and glycerol conditions. However, no match was found between the distribution of ACOF under the dry condition and any of the three continuous distributions evaluated. Based on limited data, a normal distribution might be more appropriate due to its simplicity, practicality and familiarity among the three distributions evaluated.
\end{abstract}

Keywords: Available coefficient of friction, footwear and floor interface, stochastic distribution

\section{Introduction}

The data from the Liberty Mutual Safety Index (Liberty Mutual Research Institute for Safety, 2010) showed that costs for disabling workplace injuries in 2008 due to falls on the same level in the US were estimated to be approximately 8.37 billion US dollars or $15.7 \%$ of the total cost burden. The same data also showed that the cost of falls on the same level increased by $41.9 \%$ between 1998 and 2008 after adjusting for inflation, while the overall costs of disabling workplace injuries increased only $2 \%$ over the same period.

Friction plays an important role in falls on the same level (Chang et al., 2001). The available coefficient of friction (ACOF) for human locomotion is the maximum coefficient of friction (COF) that can be supported without a slip at the shoe and floor interface. The required friction coefficient (RCOF) is defined as the minimum coefficient of friction needed at the shoe and floor interface to support hu- man locomotion. A statistical model was introduced by Chang (2004) to estimate the probability of slip incidents by comparing the ACOF with the RCOF, assuming that both coefficients have stochastic distributions. The stochastic distribution of the RCOF was recently investigated by Chang et al. (2008).

The ACOF is usually measured by mechanical devices and always has random variations even when identical samples are used (Chang and Matz, 2001). In addition, there are variations in friction with different samples of identical floor and shoe materials as shown by Chang and Matz (2001). Therefore, the ACOF for a particular interface is not a constant, but has a stochastic distribution. The concept of a distribution in the ACOF was discussed by Barnett (2002) and Marpet (2002). Barnett (2002) obtained 400 measurements of the ACOF over 100 new tiles under the dry condition with the Horizontal Pull Slipmeter (HPS) and reported that the distribution of the ACOF was near a Weibull distribution. However, most of slip incidents occur on surfaces with contamination.

${ }^{*}$ Corresponding author. E-mail: wen.chang@libertymutual.com. 
The goal of the current study was to investigate the stochastic distributions of the ACOF of quarry tiles under dry, water and glycerol contaminated conditions as an input to the statistical model.

\section{Method}

As a part of a larger study, a walkway of approximately $6.1 \mathrm{~m}$ long and $81.3 \mathrm{~cm}$ wide covered with plain quarry tiles was constructed. Fifty tiles along the walking path on this walkway were selected for friction measurements. Two friction measurements were performed on each selected tile, one in each walking direction, for a total of 100 measurements under each surface condition. The portable inclinable articulated strut slip tester (PIAST), also known as the Brungraber Mark II, is widely used in the USA and was used in this experiment. The American Society for Testing and Materials (ASTM) publishes a standard test method for this slipmeter (ASTM F1677-05, 2005).

Friction was measured under three different surface conditions: dry, water and $45 \%$ glycerol concentration. Before performing friction measurements, a clean paper towel was used to apply a solution of diluted $50 \%$ ethanol mixed with de-ionized water for cleaning the floor surfaces. To help form a uniform film over the surface, a wetting agent (Kodak Professional Photo-Flo 200 solution; Eastman Kodak Company, Rochester, NY, USA) was added to the water with 1 to 200 ratio by volume as suggested by its manufacturer. A fine water mist was applied with a garden sprayer. The process was terminated as soon as a uniform layer of water was generated on the surface judged by visual inspections. A mixture of $45 \%$ glycerol with water by weight was used as another contaminant and was applied with a paint roller. During a friction measurement on a contaminated surface, water or glycerol was replenished throughout repeated impacts on the floor surface.

Smooth Neolite test liners were used for friction measurements in this experiment. The material was cut into 7.62 by $7.62 \mathrm{~cm}$ squares to be affixed to the test foot holder of the slipmeter as a footwear test pad. The surface of the Neolite pad was sanded before the first measurement at each location under each floor surface condition. Using hand pressure, the Neolite specimen was sanded with 400 grit silicon carbide abrasive paper. The sanding process consisted of four sanding sequences of five strokes, each approximately $15 \mathrm{~cm}$ in length and always in the same di- rection on the abrasive paper. The first sanding sequence started in the direction parallel to the friction measurement. The sample was then rotated $90^{\circ}$ and the sanding sequence was repeated. The rotation and sanding of the sample were repeated two more times. The final sanding sequence was in the direction perpendicular to that of the friction measurements. Excess particles on the Neolite surface due to sanding were removed with a brush. The Neolite surface was visually inspected for scratches or dirt; the sanding procedure was repeated until these defects were removed.

According to the protocol suggested by the manufacturer, the friction measurement should start at a low coefficient of friction and increase by 0.05 as long as a non-slip persists. Once a slip occurs, the coefficient of friction is reduced by 0.01 as long as the slip persists. The measurement is stopped at the next occurrence of a non-slip. The coefficient of friction of the last slip is recorded as the result of the measurement.

An important part of operating the PIAST is to determine whether a slip occurs at the footwear and floor interface. The slip criterion used in this experiment was that only a fast movement was considered a slip as proposed by Chang (2002). Therefore, any slow movement, any finite dwell time at the impact or any partial stroke were considered non-slips. The determination of a fast movement was based on two sounds generated during a strike in the measurement process. The first sound comes from the footwear sample impacting the floor surface, and the second sound comes from the slipmeter reaching a fullstroke movement. When the operators have some experience with this slipmeter, they become very familiar with the duration between these two sounds. This helps establish the criterion of a fast movement. A slow movement has a noticeably different duration. One operator performed all the friction measurements.

For each surface condition, the distribution of the ACOF was considered as a good match with a possible continuous distribution (normal, log-normal or Weibull) with a statistical significance as determined by the Kolmogorov-Smirnov goodness-of-fit test. The Kolmogorov-Smirnov goodness-of-fit test is a nonparametric test and works for non-normally distributed data as well. 


\section{Results and Discussion}

The minimum, maximum, mean, standard deviation, skewness and kurtosis of the measured coefficient of friction for each surface condition are shown in Table 1.

Table 1

Minimum, maximum, mean, standard deviation, skewness and kurtosis of the coefficient of friction for each surface condition

\begin{tabular}{|l|c|c|c|c|c|c|}
\hline $\begin{array}{l}\text { Surface } \\
\text { Condition }\end{array}$ & Min & Max & Mean & Stdev & Skewness & Kurtosis \\
\hline Dry & 0.79 & 0.88 & 0.838 & 0.017 & -0.14 & 0.44 \\
\hline Wet & 0.26 & 0.47 & 0.350 & 0.045 & 0.43 & -0.19 \\
\hline Glycerol & 0.23 & 0.57 & 0.370 & 0.074 & 0.30 & -0.57 \\
\hline
\end{tabular}

Note: Stdev: standard deviation

The $p$ values for the Kolmogorov-Smirnov goodness-of-fit test for each surface condition are shown in Table 2. When $p>0.05$, a good match with a statistical significance exists between the distribution from the data and the particular continuous distributions. The results of the Kolmogorov-Smirnov goodness-of-fit test indicated that the ACOF appears to fit the normal and log-normal distributions better than the Weibull distribution for the water and glycerol conditions. However, no match was found between the distribution of ACOF under the dry condition and any of the three continuous distributions evaluated.

Table 2

The $p$ values for the Kolmogorov-Smirnov goodness-of-fit test for each surface condition

\begin{tabular}{|l|c|c|c|}
\hline $\begin{array}{l}\text { Surface } \\
\text { Condition }\end{array}$ & Normal & Log-normal & Weibull \\
\hline Dry & $<0.01$ & $<0.01$ & $<0.001$ \\
\hline Wet & $\mathbf{0 . 0 7 7}$ & $\mathbf{0 . 0 7 5}$ & 0.047 \\
\hline Glycerol & $\mathbf{0 . 1 3 6}$ & $\mathbf{0 . 0 8}$ & $\mathbf{0 . 0 9 3}$ \\
\hline
\end{tabular}

The distributions of the data for each surface condition are shown with the three continuous distributions in Figures 1, 2 and 3 for dry, water and $45 \%$ glycerol conditions, respectively.

The distributions of the RCOF were investigated by Chang et al. (2010). Their results indicated that the RCOF distribution for each individual under each walking condition had a slightly larger number of cases that matched a normal distribution than the lognormal and Weibull distributions. Therefore, the normal distribution was a preferred representation for the RCOF due to its simplicity, practicality and familiarity among three distributions evaluated. However, there were a few cases in which none of the three distributions was a good match for the RCOF.

Similar results were found for the ACOF. Although both the normal and log-normal distributions had a good match with the ACOF, the normal distribution could be used to represent the ACOF distribution due to its simplicity, practicality and familiarity compared with the log-normal distribution.

\section{Conclusions}

Based on limited data, a normal distribution might be more appropriate due to its simplicity, practicality and familiarity among three distributions evaluated. However, no match was found for the dry conditions. More measurements are needed to explore other surfaces and conditions.

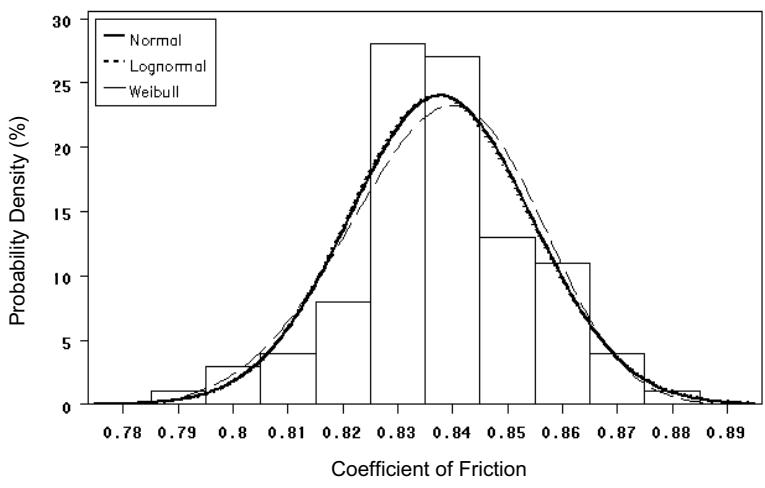

Figure 1 The stochastic distribution of the ACOF for dry conditions

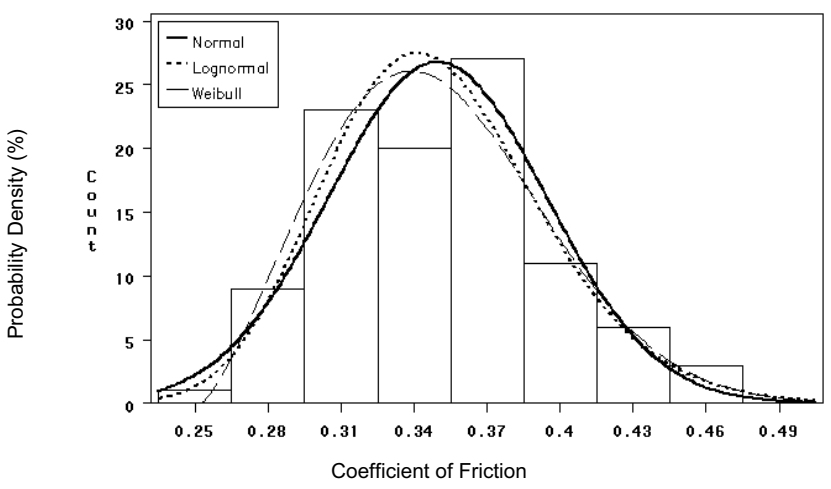

Figure 2 The stochastic distribution of the ACOF for water conditions 


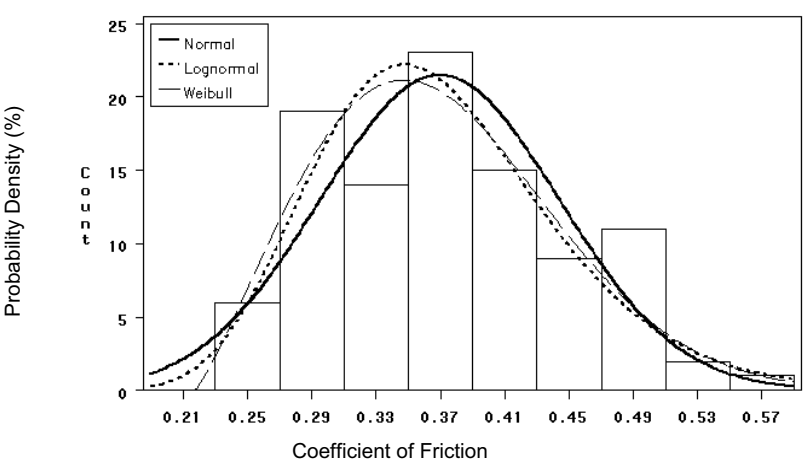

Figure 3 The stochastic distribution of the ACOF for $45 \%$ glycerol conditions

\section{References}

American Society for Testing and Materials, 2005, F-1677-05 Standard method of test for using a portable inclineable articulated strut slip tester (PIAST), Annual Book of ASTM Standards, Philadelphia, American Society for Testing and Materials.
Barnett, R. L., 2002, Slip and fall theory - Extreme order statistics, International Journal of Occupational Safety and Ergonomics, $8(2), 135-159$.

Chang, W. R., 2002, The effects of slip criterion and time on friction measurements, Safety Science, 40 (7-8), 593-611.

Chang, W. R., 2004, A statistical model to estimate the probability of slip and fall incidents, Safety Science, 42 (9), 779-789.

Chang, W. R., and Matz S., 2001, The slip resistance of common footwear materials measured with two slipmeters, Applied Ergonomics, 32 (6), 549-558.

Chang, W. R., Grönqvist, R., Leclercq, S., Myung, R., Makkonen, L., Strandberg, L., Brungraber, R., Mattke, U. and Thorpe, S., 2001, The role of friction in the measurement of slipperiness, Part 1: Friction mechanisms and definition of test conditions, Ergonomics, 44 (13), 1217-1232.

Chang, W. R., Chang, C. C., Matz, S. and Lesch, M. F., 2008, A methodology to quantify the stochastic distribution of friction coefficient required for level walking, Applied Ergonomics, 39 (6), 766-771.

Chang, W. R., Matz, S. and Chang, C. C., 2010, The stochastic distribution of required coefficient of friction for level walking, The Proceedings of the Sixth World Congress of Biomechanics, Singapore, August 1 - 6.

Liberty Mutual Research Institute for Safety, 2010, 2010 Workplace Safety Index. From Research to Reality, 13 (3) available at http://www.libertymutual.com/researchinstitute.

Marpet, M. I., 2002, Improved characterization of tribometric test results, Safety Science, 40 (7-8), 705-714. 